\title{
Analysis of the Noise Reduction Characteristics of a Diffuser with Fizeau Interferometry
}

\author{
Hongjun Wang, Xueliang Zhu $\mathbb{D}^{D}$, Bingcai Liu $\mathbb{D}^{D}$, and Ailing Tian $(\mathbb{D}$ \\ Xi'an Technological University, International Center for Optical Research and Education (iCORE), No. 2 Xuefu Middle Road, \\ Xi'an, Shaanxi 710021, China \\ Correspondence should be addressed to Xueliang Zhu; zhuxueliang@xatu.edu.cn
}

Received 20 August 2020; Revised 12 January 2021; Accepted 17 February 2021; Published 23 February 2021

Academic Editor: Adrian Podoleanu

Copyright (c) 2021 Hongjun Wang et al. This is an open access article distributed under the Creative Commons Attribution License, which permits unrestricted use, distribution, and reproduction in any medium, provided the original work is properly cited.

\begin{abstract}
To solve the coherent noise problem of an interference image, the method of a rotating diffuser was adopted to change the coherence of a beam to reduce the noise of the interference system. The relationship between the speed of the diffuser and the signal-to-noise ratio (SNR) of the fringe contrast system was simulated to obtain the diffuser control parameters needed for the best interference fringe state. The fringe contrast of each image and the SNR of the system were analyzed. The results showed that the increased speed of the diffuser reduced the contrast of the interference image to a certain extent, but the increased speed also effectively improved the SNR and facilitated the subsequent interference image processing. Due to the coherent noise in the interferometric system, the method of the rotated diffuser reduced the coherence of the light beam to suppress the noise of the interference image. By analyzing the coherent noise reduction characteristics of the rotated diffuser with different surface roughnesses, the relationship between the surface roughness and the noise contrast for different rotation speeds was simulated, and the effective roughness range with the noise reduction effect was selected. A noise reduction system was built based on Fizeau interference, and the noise contrast of the interference image was collected and calculated. The effective range of $\sigma_{\mathrm{h}} / \lambda$ was $0.2-0.5$ when the rotation speed was $10 \mathrm{r} / \mathrm{s}$, while the effective range of $\sigma_{\mathrm{h}} / \lambda$ was $0.4-0.6$ when the rotation speed was $100 \mathrm{r} / \mathrm{s}$. The experimental results showed that the surface roughness and wavelength ratio $\sigma_{\mathrm{h}} / \lambda$ of the rotated diffuser increased when the noise contrast tended toward 1 , but the effective range of the surface roughness decreased with the increase of the rotational speed of the diffuser.
\end{abstract}

\section{Introduction}

In an interference system, the defects on the surface of the optical element (such as depression, bubbles, dust, and scars) are a new light source. As a laser has a high degree of coherence, a large amount of coherent noise is generated by the light source formed by defects, which creates Newton rings in the interferogram, thus affecting the quality of the interferogram and further affecting the interference image processing, forming a measurement error $[1,2]$. The coherent noise that affects the interference result is the intrinsic noise of the system. The suppression of intrinsic noise is a difficult problem in interferometer design. Therefore, to suppress intrinsic noise, many efforts have been made by experts and scholars over the years. For example, multiple fiber coupling is used to produce phase changes, optical wedges are used to change optical paths, and moving scatter or phase shifters are used to reduce laser coherence [3, 4]. In 1970, Asakura [5] studied the spatial coherence of a laser passing through a rotating ground glass. In 1980, Kwon et al. [6] studied the relationship between the contrast of infrared interference fringes and surface roughness. In 1983, Harwalkar [7] et al. carried out theoretical and experimental research on the scattered light passing through rotating ground glass. Chen [8] used the phase-shifting algorithm to calculate infrared interference fringe contrast and verified the theoretical relationship between the fringe contrast and the surface roughness. In 2000, Ziraki et al. [9] applied a rotating diffuser to a holographic system to suppress speckle noise. Pitter et al. [10] used a rotating diffuser for heterodyne 
interference microscopy to suppress coherent noise in coherent whole-field microscope images in 2004. Morris et al. [11] added a rotating diffuser in an interference system and then effectively reduced the coherence of the light source in 2010. In 2011, Xu et al. [12] and others calculated the statistical characteristics of the rotating ground glass in an interference system. Chen et al. [13] studied the use of a rotating diffuser in projection systems to reduce speckle distortion in 2012. In 2014, Qiao et al. [14] studied the denoising effect of rotating ground glass in a holographic system. In 2015, Wang Sen and others studied the effect of the rotational speed of ground glass on the imaging quality of a thermal ghost. These results showed that the intrinsic noise of the system could be reduced and the SNR of the system could be improved by using the rotating ground glass.

In this research, the noise reduction performance of the diffuser in a large-diameter Fizeau interferometer was mainly analyzed to improve the SNR of the interference image and improve the quality of the interference image. The optical modulation theory of diffuser was studied, included the theory of modulation of rough surface and rotation speed, and simulated the effect of surface roughness and rotation speed on the noise suppression effect of the interference image. The system of noise suppression based on Fizeau interference was built. According to the surface roughness and rotation speed of the diffuser, the noise suppression results of the interference image were verified. The noise contrast and image SNR were compared with the simulation results.

\section{Theoretical Analysis}

2.1. Fizeau Interferometer. To eliminate coherent noises, the rotating diffuser was placed near the focal plane of the imaging system to modulate the interference beam that entered the imaging system, as shown in Figure 1. The terms $\mathrm{RF}$ and TF shown represent the reference mirror and the test mirror, respectively. The light emitted by the laser passed through the spatial filter to form a point light source and then through the beam expander to form a parallel light. After the parallel light passed through the reference mirror and the test mirror, an interference pattern was formed on the CCD.

2.2. The Influence of the Diffuser Rotation Speed on the Interference Pattern. It was assumed that the incident wavefront of diffuser was $u_{0}(x, y)$, the phase introduced by the diffuser was $\Phi_{d}$, and the diffuser rotated around $\mathrm{O}$ at the angular velocity $\omega$. Then, at time $t$, the wavefront $u(x, y, t)$ could be expressed as

$$
u(x, y, t)=u_{0}(x, y) \exp \left[i \Phi_{d}\left(r, \theta_{0}-\omega t\right)\right] .
$$

In the equation, $r$ is the distance between the center of rotation and the point of incidence of light and $\theta_{0}$ is the initial angle of rotation of the diffuser. The spot position was as shown in Figure 2. During the measuring process, $r$ and $\omega$ were constants. Then, $\exp \left[i \Phi_{d}\left(r, \theta_{0}-\omega t\right)\right]$ was just a function of the time $t$ in equation (1).

It was assumed that the imaging lens magnification was 1. Then, the light field $U\left(x_{1}, y_{1}, t\right)$ on the CCD was expressed with

$$
U\left(x_{1}, y_{1}, t\right)=\int_{-\infty}^{\infty} \int_{-\infty} k\left(x+x_{1}, y+y_{1}\right) u(x, y, t) \mathrm{d} x \mathrm{~d} y
$$

In the equation, $k(x, y)$ is the amplitude point spread function of an imaging system. According to the speckle interference theory, the coherent time of the speckle, $\tau_{c}$ [15], is

$$
\tau_{c}=\frac{8 \lambda z}{3 \pi^{2} \omega \mathrm{rD}}
$$

In the equation, $z$ is the distance between the diffuser and the pupil of the imaging system, $D$ is the entrance pupil diameter, and $\lambda$ is the wavelength. Within the exposure time $T$ of the CCD, there are $N=T / \tau_{c}$ different speckle images which could be acquired. When $N$ was large enough, the contrast of the speckle, $C_{n}[15]$, was

$$
C_{n} \propto \frac{1}{\sqrt{N}}=\sqrt{\frac{8 \lambda z}{3 \pi^{2} \omega \mathrm{rDT}}}
$$

According to the interference theory, the contrast of the interference fringes, $C_{s}[15]$, was

$$
C_{s} \propto \sin c\left(\frac{\tau_{R T}}{\tau_{c}}\right)=\sin c\left(\frac{3 \pi^{2} \omega \mathrm{rD} \tau_{\mathrm{RT}}}{8 \lambda z}\right) .
$$

In the equation, $\tau_{\mathrm{RT}}$ is the time delay of the test light and the reference light. The ratio of the interference fringe contrast to the speckle contrast was defined as the signal-to-noise ratio (SNR) of the interference system. Therefore, the SNR of the interference system with a rotating diffuser could be written as

$$
\mathrm{SNR}=\frac{C_{s}}{C_{n}} \propto \frac{\sin c\left(3 \pi^{2} \omega \mathrm{rD} \tau_{R T} / 8 \lambda z\right)}{\sqrt{8 \lambda z / 3 \pi^{2} \omega \mathrm{rDT}}} .
$$

From equation (6), it could be seen that the SNR of the interference system was related to the rotational angular velocity of the diffuser, the exposure time of the detector, and the delay time of the different light path.

2.3. The Influence of the Diffuser Surface Roughness on the Interference Pattern. To study the corresponding relationship between the noise contrast and the surface roughness of diffuser, it was assumed that the scattering types were surface scattering, as shown in Figure 3.

Figure 4 shows a reflection light path diagram in a free space, for which $\widehat{n}$ is a unit vector indicating the direction of the average normal surface, $\hat{i}$ is a unit vector representing the direction of the incident light, and $\widehat{o}$ is the unit vector showing the direction of the observation point on the right plane. 
When the light was perpendicularly incident on the surface of the diffuser, at any point on the surface, the phase delay $\Phi(\alpha, \beta)$ associated with propagating to the surface and scattering from the surface was as follows [15]:

$$
\Phi(\alpha, \beta)=\frac{2 \pi}{\lambda}[1+(\widehat{o} \cdot \widehat{n})] h(\alpha, \beta),
$$

where $\widehat{n}$ is the normal outward of the surface and $\widehat{o}$ is the unit vector pointing to the observation point. For paraxial imaging, $\widehat{o} \cdot \widehat{n} \cong 1$, equation (7) could be simplified to

$$
\Phi(\alpha, \beta)=\frac{4 \pi}{\lambda} h(\alpha, \beta) .
$$

It was assumed that the incident intensity was 1 and the light transmittance was 1 , so the outgoing light $a(\alpha, \beta)$ depended on the distribution of the rough surface and the expression was as follows:

$$
a(\alpha, \beta)=\exp [j \Phi(\alpha, \beta)]=\exp \left[j \frac{4 \pi}{\lambda} h(\alpha, \beta)\right] .
$$

If the focal distances of the two lenses were the same, the magnification of the imaging system was 1 , and the image $A(x, y)$ in the $(x, y)$ coordinate could be written as a convolution:

$$
A(x, y)=\int_{-\infty}^{\infty} \int_{-\infty} h(x-\alpha, y-\beta) a(\alpha, \beta) \mathrm{d} \alpha \mathrm{d} \beta .
$$

The relationship between the point spread function $h(\alpha, \beta)$ and the pupil function $P(\xi, \eta)$ of the imaging system was

$$
h(\alpha, \beta)=\frac{1}{\lambda \cdot f^{2}} \int_{-\infty}^{\infty} \int_{-\infty} P(\xi, \eta) \exp \left[j \cdot \frac{2 \pi}{\lambda \cdot f}(\alpha \xi+\beta \eta)\right] \mathrm{d} \xi \mathrm{d} \eta .
$$

It was assumed that the system had no aberrations, so $P$ $(\xi, \eta)=p(\xi, \eta)$ was a real value. It was also assumed that $p$ was 1 at the origin and the point spread function $h$ was a real value.

The relationship between the surface roughness $\sigma$ and the noise contrast $C$ could be obtained as follows:

$$
C=\sqrt{\frac{8(N-1) \cdot\left\{N-1+\cosh \left(\sigma_{\phi}^{2}\right)\right\} \sinh \left(\sigma_{\phi}^{2} / 2\right)}{N\left[N-1+\exp \left(\sigma_{\phi}^{2}\right)\right]^{2}} .}
$$

Based on equation (12), it could be seen that the different surface roughnesses of the diffuser had different influences on the noise contrast. We knew that the diffuser needed to be in the state of rotation to play a role in noise suppression, but the above theory did not consider the rotation speed, so the effects of different surface roughnesses on the noise suppression were analyzed for different rotation speeds, as described next.

It was assumed that the complex amplitude $\alpha(\alpha, \beta)$ of the scattered wave above the surface was related to the surface height through a purely geometrically similar relationship. A phase was added to $\alpha$ that represented the phase delay that spread the surface and scattered from the surface:

$$
a(\alpha, \beta)=r S(\alpha, \beta) e^{j \Phi(\alpha, \beta)},
$$

where $r$ denotes the average amplitude reflectivity of the surface and $S(\alpha, \beta)$ indicates the complex amplitude of the illumination over the entire scattered spot and $\Phi$ represents the dot product in the expression, which was

$$
\Phi(\alpha, \beta)=\frac{2 \pi}{\lambda}(-\hat{i} \cdot \widehat{n}+\widehat{o} \cdot \widehat{n}) \cdot h(\alpha, \beta) .
$$

When the illumination direction or observation direction deviated from the surface normal, the height fluctuation of the surface decreased due to the perspective relationship. The relationship between the variance $\sigma_{\phi}^{2}$ of the phase shift and the variance $\sigma_{h}^{2}$ of the surface height fluctuation was

$$
\sigma_{\phi}^{2}=\left[\frac{2 \pi}{\lambda}(-\hat{i} \cdot \widehat{n}+\widehat{o} \cdot \widehat{n})\right]^{2} \sigma_{h}^{2}
$$

The relationship between the phase shift correlation function $\Gamma \Phi(\Delta \alpha, \Delta \beta)$ and the normalized correlation function $\mu_{h}(\Delta \alpha, \Delta \beta)$ of the surface height fluctuation was

$$
\Gamma_{\Phi}(\Delta \alpha, \Delta \beta)=\sigma_{\phi}^{2} \mu_{h}(\Delta \alpha, \Delta \beta) .
$$

The normalized autocorrelation function of the light field was

$$
\mu_{\alpha}(\Delta \alpha, \Delta \beta)=\overline{\exp \left[j\left(\varphi_{1}-\varphi_{2}\right)\right]} .
$$

Then, a mathematical hypothesis was made about the relative function $\mu_{h}$ of the surface height fluctuations:

$$
\mu_{h}(\Delta \alpha, \Delta \beta)=\exp \left[-\left(\frac{r}{r_{c}}\right)^{2}\right],
$$

where $r=\sqrt{\Delta \alpha^{2}+\Delta \beta^{2}}$, and $r_{c}$ is the radius of the normalized surface correlation down to $1 /$ e. Therefore,

$$
\mu_{\alpha}(\Delta \alpha, \Delta \beta)=\exp \left\{-\sigma_{\varphi}^{2}\left\{1-\exp \left[-\left(\frac{r}{r_{c}}\right)^{2}\right]\right\}\right\} \text {. }
$$

The surface height of the diffuser was fluctuant, and $r$ was the displacement of the coherent noise. When the diffuser rotated, the coherent noise displacement was equal to the product of the rotating speed of the diffuser and the exposure time; that is, $r=\mathrm{vt}$, as shown in Figure 5. By substituting $r=\mathrm{vt}$ into equation (19), the following expression could be obtained:

$$
\mu_{\alpha}(\Delta \alpha, \Delta \beta)=\exp \left\{-\sigma_{\varphi}^{2}\left\{1-\exp \left[-\left(\frac{\mathrm{vt}}{r_{c}}\right)^{2}\right]\right\}\right\} .
$$

The spatial coherent area $A_{\alpha}$ of the nonspecular reflection components was

$$
A_{\alpha}=2 \pi \int_{0}^{\infty} v^{2} t \mu_{\alpha}(t) \mathrm{d} t
$$

Hence, 


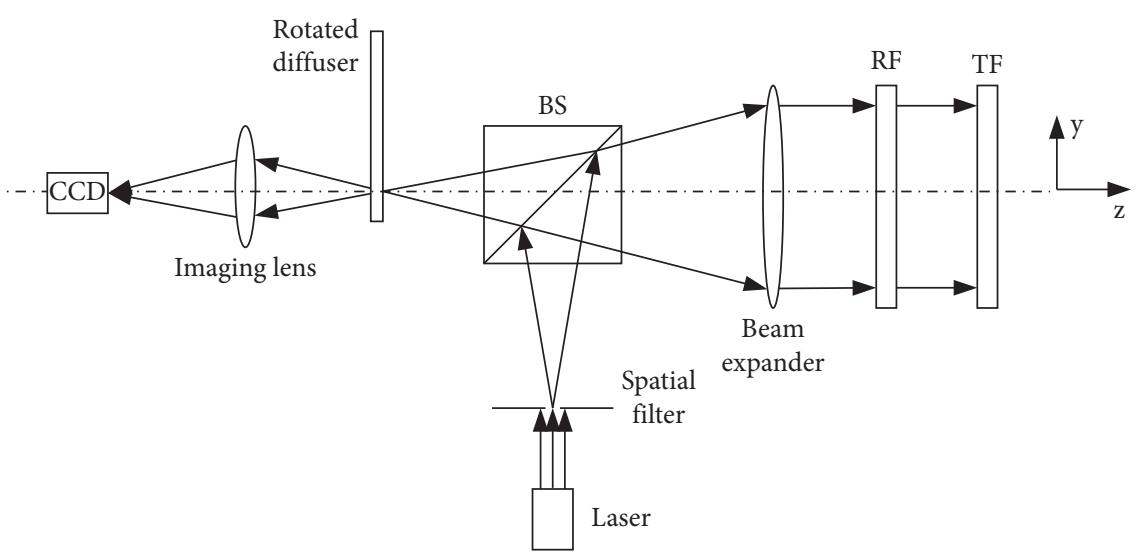

FIGURE 1: Fizeau interferometer with rotated diffuser.

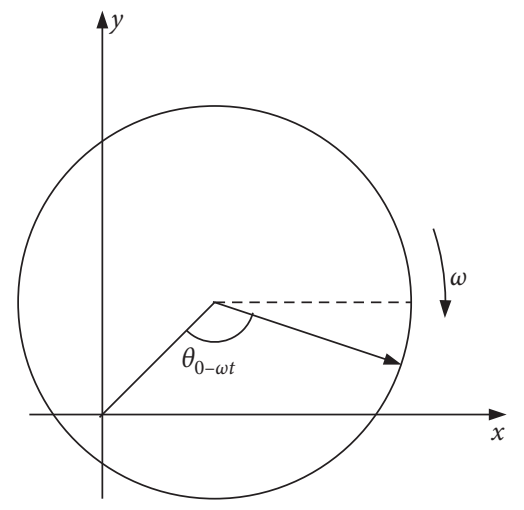

FIGURE 2: Spot position.

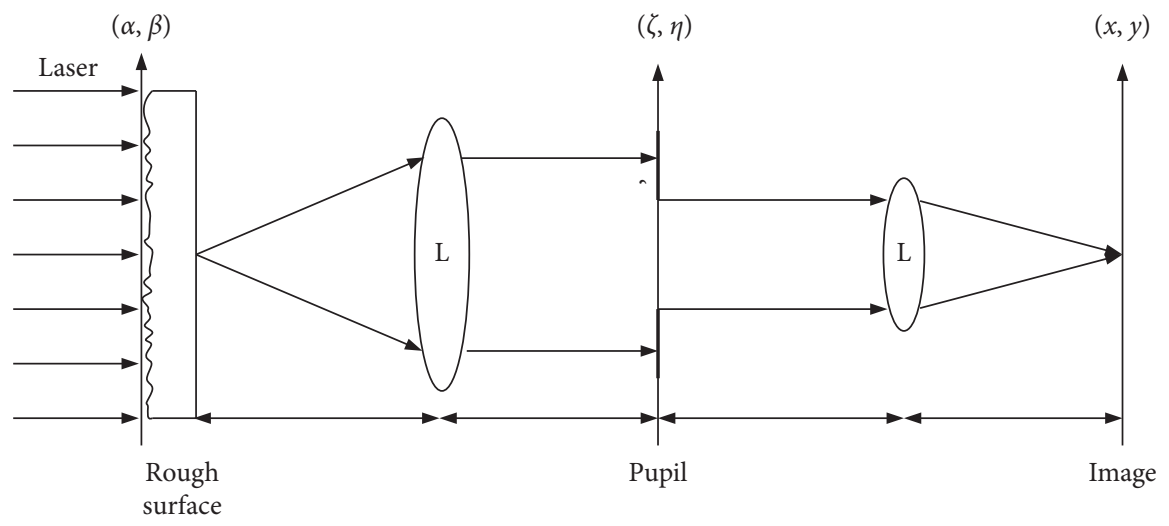

FIGURE 3: Imaging light path diagram for rough surface noise suppression. 

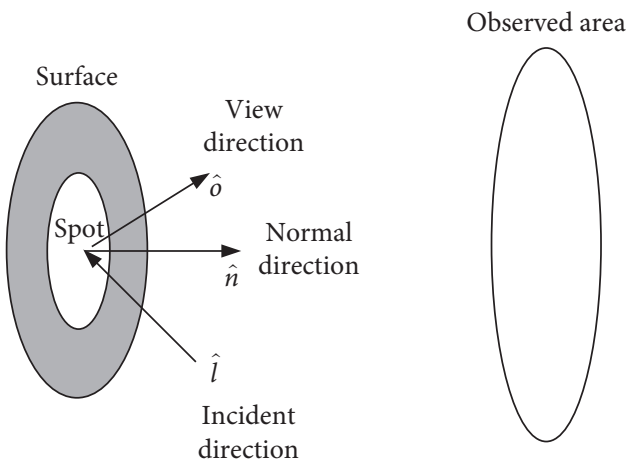

FIgURE 4: Free space reflection light path diagram [15].

$$
\begin{aligned}
A_{\alpha}=\frac{2 \pi e^{-\sigma(2 / \varphi)}}{1-e^{-\sigma_{\varphi}^{2}}} \int_{0}^{\infty} & v^{2} t\left[\exp \left(\sigma_{\varphi}^{2} \exp \left[\left(v_{t} / r_{c}\right)^{2}\right] 1\right] \mathrm{d} t\right. \\
& =\frac{\pi e^{-\sigma_{\varphi}^{2}}}{1-e^{-\sigma_{\varphi}^{2}}} \frac{\left[\operatorname{Ei}\left(\sigma_{\varphi}^{2}\right) \varepsilon \ln \left(\sigma_{\varphi}^{2}\right)\right]}{V^{2}} .
\end{aligned}
$$

Here, Ei $(x)$ is the exponential integral function and $\varepsilon$ is the Euler constant. The number $N^{\prime}$ of these completely incoherent noise fields is the ratio of the spatial coherence area $A_{k}$ and the total illumination area $A_{\alpha}$; it was found that

$$
N^{\prime}=\frac{v^{2} N_{0}\left(e^{\sigma_{\phi}^{2}}-1\right)}{E i\left(e^{\sigma_{\phi}^{2}}\right)-\varepsilon-\ln \left(\sigma_{\phi}^{2}\right)} .
$$

Bringing equation (23) into equation (12) produced a new numerical relationship between the surface roughness of the diffuser and the noise contrast:

$$
C=\sqrt{\frac{8\left(N^{\prime}-1\right)\left\{N^{\prime}-1+\cosh \left(\sigma_{\varphi}^{2}\right)\right\} \sinh \left(\sigma_{\varphi}^{2} / 2\right)}{N^{\prime}\left[N^{\prime}-1+\exp \left(\sigma_{\varphi}^{2}\right)\right]^{2}} .}
$$

\section{Simulation}

3.1. System Parameter. According to the measurement principle, the Fizeau interference experiment system was built. The time delay $\tau_{\mathrm{RT}}$ between the testing light path and the reference light path was $4.76 \times 10^{-9} s$, the exposure time $T$ of the CCD was $1 / 714 \mathrm{~s}$, the pupil diameter $D$ was $7 \mathrm{~mm}$, the wavelength $\lambda$ was $650 \mathrm{~nm}$, and the distance $r$ between the center of rotation and the diameter of the incidence light was $20 \mathrm{~mm}$.

3.2. Relationship between the Rotation Speed and the Image Quality. The relationship between the rotation speed of the diffuser and the coherent time could be calculated according to equation (3), as shown in Figure 6(a). As can be seen from Figure 6(a), the coherent time decreased gradually as the rotational speed of the diffuser increased, and the falling speed slowed down with the increase of the rotational speed of the diffuser. According to equation (6), the relationship between the rotation speed of the diffuser, the contrast of the fringe and speckle, and the signal-to-noise ratio of the system could be simulated, as shown in Figures 6(b)-6(d). Figure 6(b) shows that the image SNR was improved with the increase of the diffuser speed. When the diffuser speed was in the $40 \mathrm{r} / \mathrm{s}-90 \mathrm{r} / \mathrm{s}$, fringe contrast was between 0.999-0.994, the relative value of decline was not obvious, and more fringe information was retained. At the same time, the relative value of the speckle contrast was between 0.5 and 0.3. Compared with the relative value of the fringe contrast, the decrease trend was obvious, indicating that properly increasing the rotational speed of diffuser could effectively suppress the coherent noise of the interference image.

\subsection{Relationship between the Rotation Speed of the Diffuser} and the Image Quality. Equation (24) describes the effect of the different surface roughnesses on the coherent noise contrast at different rotation speeds when $N_{0}$ was equal to 5 , 10,100 , and 1000 . The effect of different $N_{O}$ to noise contrasts at different rotation speeds was simulated in the range of $\sigma_{\mathrm{h}} / \lambda$ (surface height standard deviation of wavelength normalization) equal to $0.2-0.68$. The simulated relationship curves are shown in Figures 7 and 8.

Figure 7 shows the relationship between the noise contrast $\mathrm{C}$ and the surface roughness at different rotational speed $v$ and $\mathrm{N}_{0}$. It can be seen from the figure that (1) for an ideally flat surface $\left(\sigma_{\mathrm{h}} / \lambda=0\right)$, the noise contrast was 0 ; (2) as $\sigma_{\mathrm{h}} / \lambda$ increased, the contrast was finally saturated at 1 ; and ( 3 ) as the rotational speed of the diffuser increased, the surface roughness corresponding to the saturated contrast also increased.

The relationship between the noise contrast $\mathrm{C}$ and $N_{0}$, different rotation frequencies $v$, and $\sigma_{\mathrm{h}} / \lambda$ for different diffusers is shown in Figure 8. It can be seen from the figure that for a small roughness value, the contrast $\mathrm{C}$ first increased with the parameter $\mathrm{N}_{0}$ and then finally approached zero continuously. This was caused by the different dependence of the mirror reflection component and the diffuser component of light on the value of $\mathrm{N}_{0}$. Especially in the light path, the mirror reflection component in the reflected light always appeared in the form of a small light spot on the optical axis in the focal plane of the first lens, while the diffuser light filled the entire pupil. It was necessary to change the size of the pupil to change $\mathrm{N}_{0}$ and to decrease the pupil to increase $\mathrm{N}_{0}$. As the mirror reflection component was always limited to the optical axis region, the mirror reflection component was not affected when the diameter of the pupil was reduced. However, the average intensity of the scattered light that was transmitted decreased in proportion to the pupil area as the pupil shrank. Therefore, when the value of $\sigma_{\mathrm{h}} / \lambda$ was small, $\mathrm{N}_{0}$ increased from 1 or more so that the noise contrast increased from zero. Additionally, when $\mathrm{N}_{0}$ finally increased further, the $\sigma_{\mathrm{I}}$ decreased and $\bar{I}$ remained constant, so the contrast decreased. Finally, when the pupil 


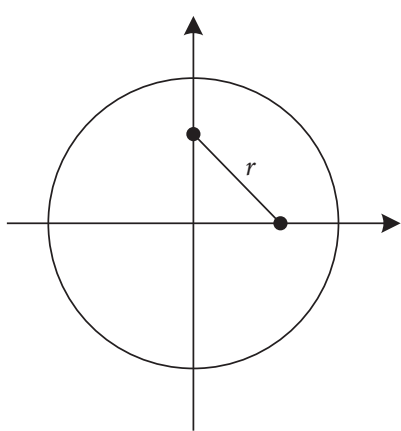

(a)

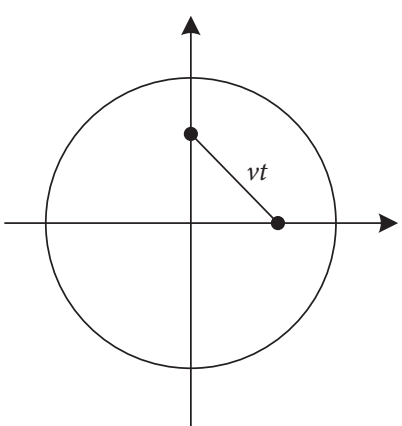

(b)

FIGURE 5: Displacement of the coherent noise.

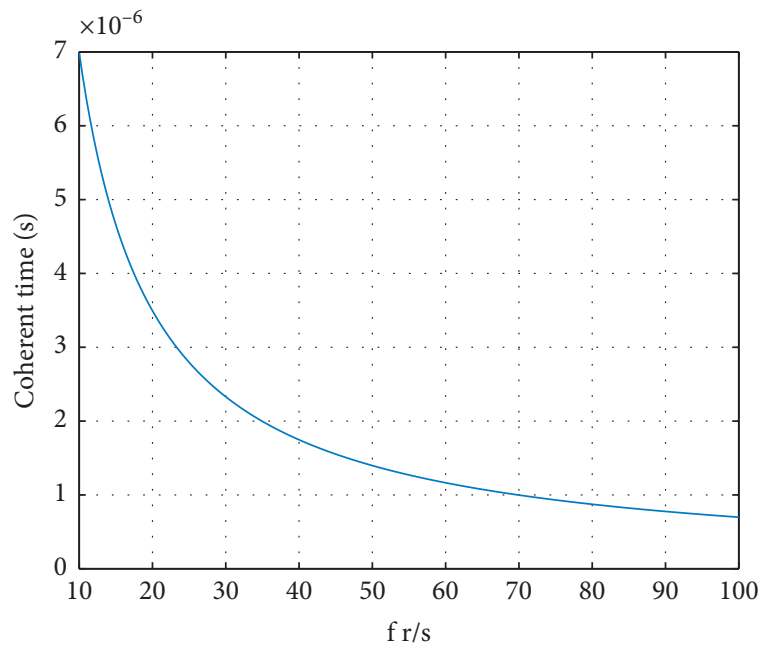

(a)

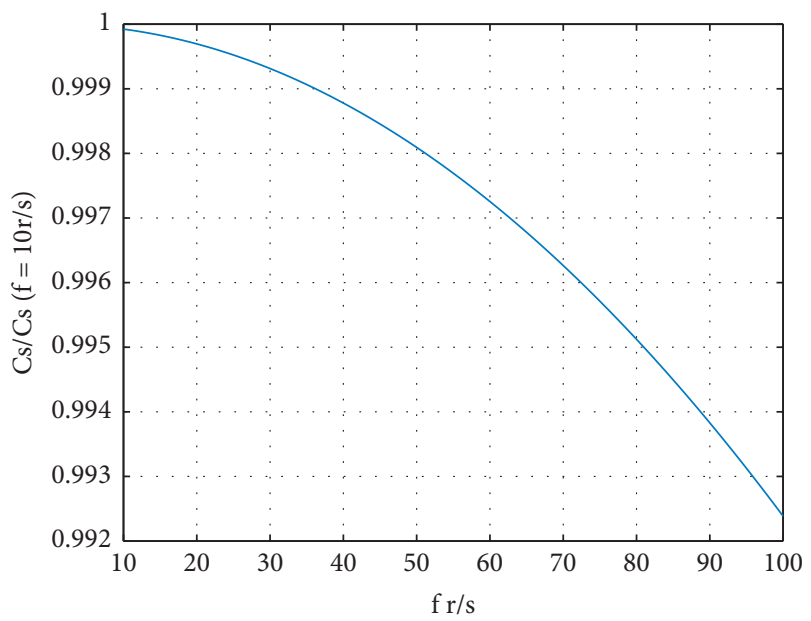

(c)

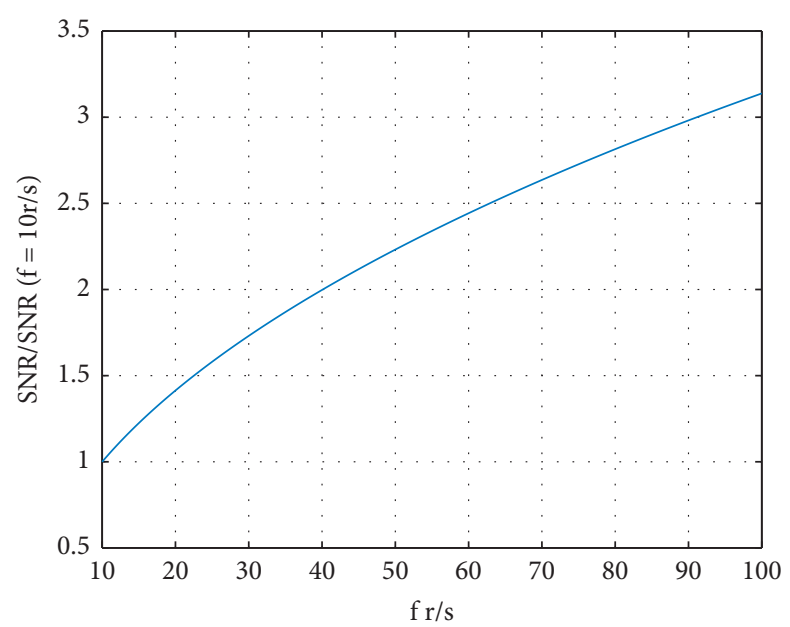

(b)

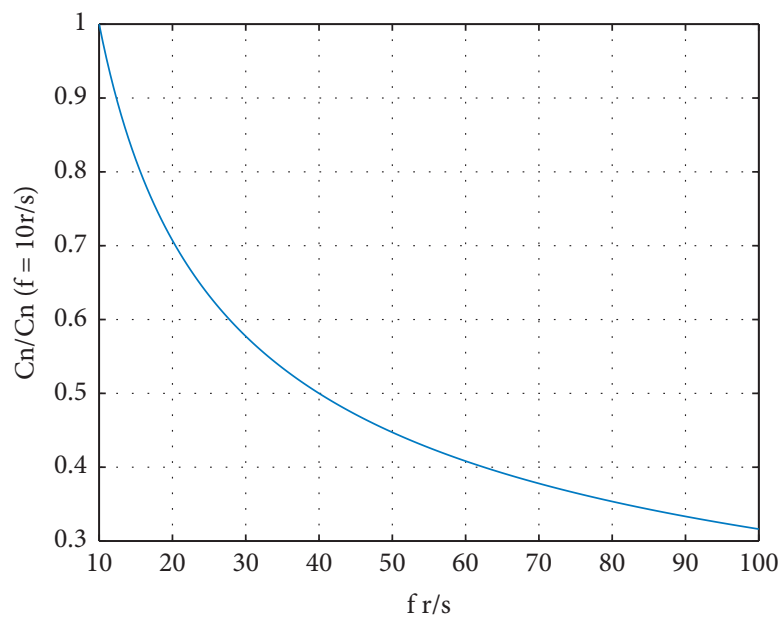

(d)

FIgURE 6: The influence of the rotation frequency on the image quality. (a) The influence on the coherent time. (b) The influence on the SNR. (c) The influence on the contrast of the fringes. (d) The influence on the speckle contrast. 


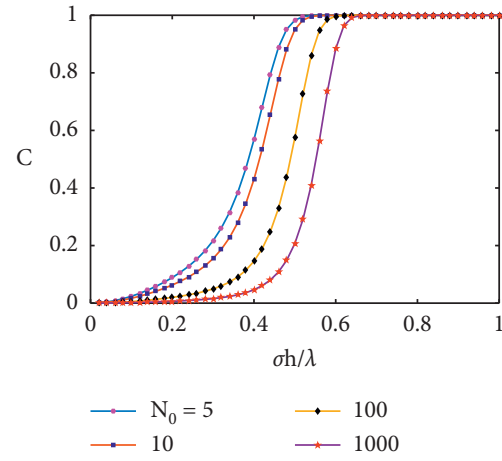

(a)

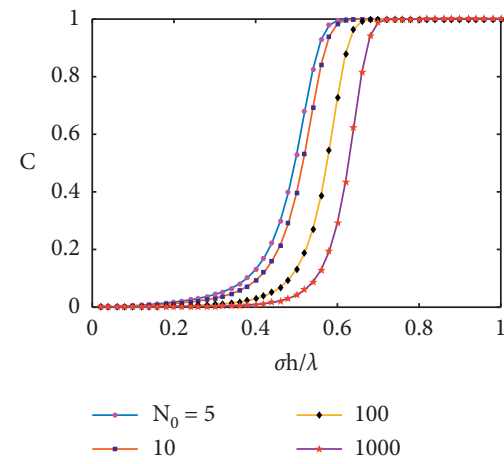

(b)

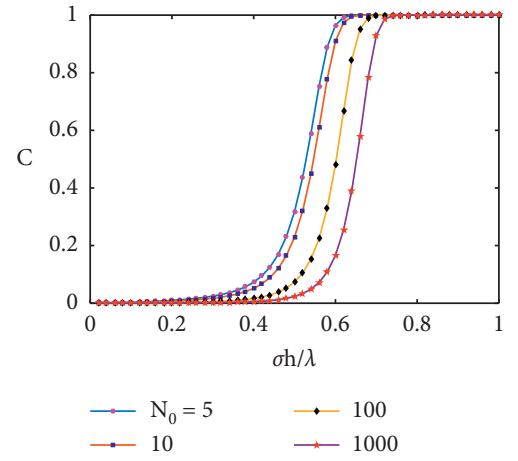

(c)

Figure 7: The relationship between the contrast $\mathrm{C}$ and $\sigma_{\mathrm{h}} / \lambda$ at different speeds $v$ and $\mathrm{N}_{0}$. (a) $\mathrm{V}=10 \mathrm{r} / \mathrm{s} .(\mathrm{b}) \mathrm{V}=50 \mathrm{r} / \mathrm{s}$. (c) $\mathrm{V}=90 \mathrm{r} / \mathrm{s}$.

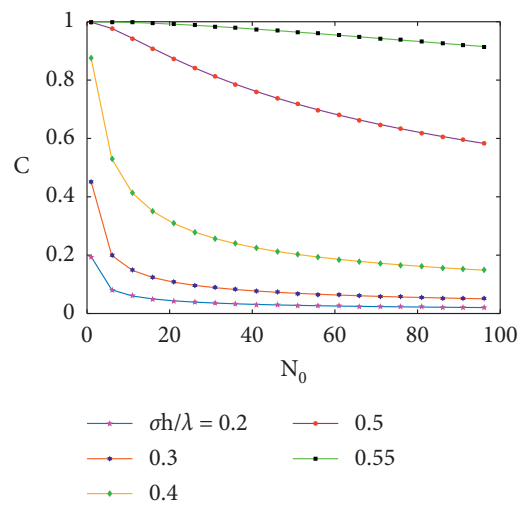

(a)

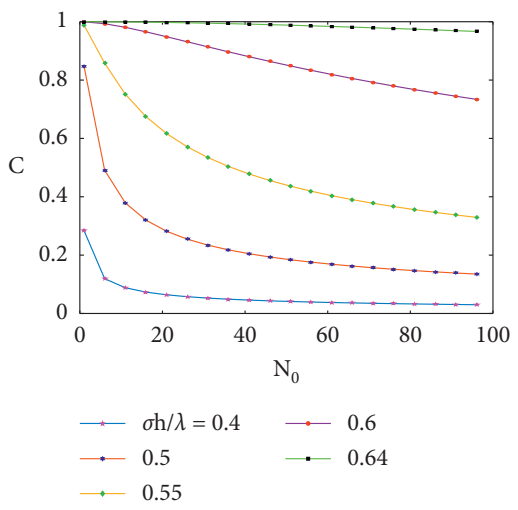

(b)

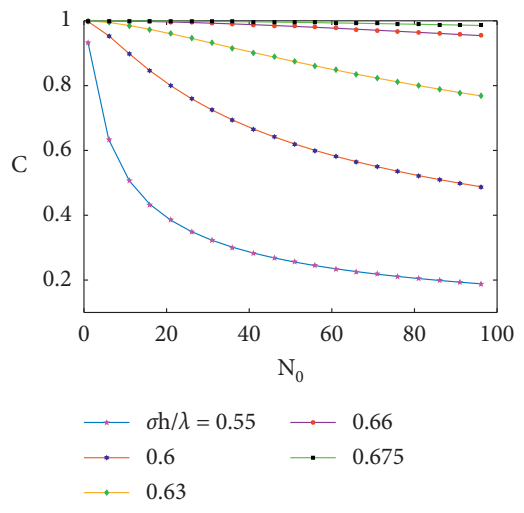

(c)

Figure 8: The relationship between the contrast $\mathrm{C}$ and $\mathrm{N}_{0}$ at different speeds $v$ and $\sigma_{\mathrm{h}} / \lambda$. (a) $\mathrm{V}=10 \mathrm{r} / \mathrm{s}$. (b) $\mathrm{V}=50 \mathrm{r} / \mathrm{s}$. (c) $\mathrm{V}=90 \mathrm{r} / \mathrm{s}$.

size was small enough, both the scattered light and the reflected light were affected, and the noise contrast $\mathrm{C}$ did not fall with $\mathrm{N}_{0}$.

\section{Experimental Study}

As shown in Figure 9, in the constructed Fizeau interference system, the diffuser was placed near the focus of the imaging system, and its roughness was $0.3668 \mu \mathrm{m}$. Figure 10 shows the diffuser used in the experiment. The diameter of the diffuser was $50 \mathrm{~mm}$, and the thickness was $3 \mathrm{~mm}$.

To test different surface roughnesses of the diffuser for the noise suppression effect, a series of different roughnesses of the diffuser was used. The roughness of the diffusers was measured by Taylor Hobson PGI Optics Profilometer, as shown in Table 1.

Based on the noise estimation processing, for the case of the known surface roughness, for different speeds of the diffuser, the fringe contrast, speckle contrast, image contrast, and image SNR were as shown in Figure 11. As can be seen from Figure 11, as the rotational speed of diffuser gradually increased, the fringe contrast, speckle contrast, and image contrast of the interference image all showed a downward trend, while the image signal-to-noise ratio increased. It was also shown that the rotating diffuser could reduce the contrast of the interference image while suppressing the coherent noise but improving the signal-to-noise ratio of the image. The interference images at different rotational speeds of the diffuser are shown in Figure 12. It can be seen from the figure that with the increase of the rotational speed of the diffuser, although the contrast of the image was reduced to a certain extent, the signal-to-noise ratio of the image was improved and the coherent noise was effectively suppressed.

For the condition of invariance in the diffuser surface roughness, the rotation speed of the motor was adjusted, and the interference images were acquired for different rotation speeds. Due to the need to analyze the influence of different surface roughnesses on the noise contrast, the intervention had to be separate. The Wiener filtering method was used to separate the image noise, and then the noise contrast for different rotation speeds was as shown in Figure 13.

As can be seen from Figure 13, the roughness of the diffuser could effectively suppress the noise increases with the increase of the rotation speed of the diffuser, but the effective range of the roughness of the surface that could effectively suppress noise gradually decreased, which was highly consistent with the simulation results. When the rotation speed was $10 \mathrm{r} / \mathrm{s}$, the effective range of $\sigma_{h} / \lambda$ was 


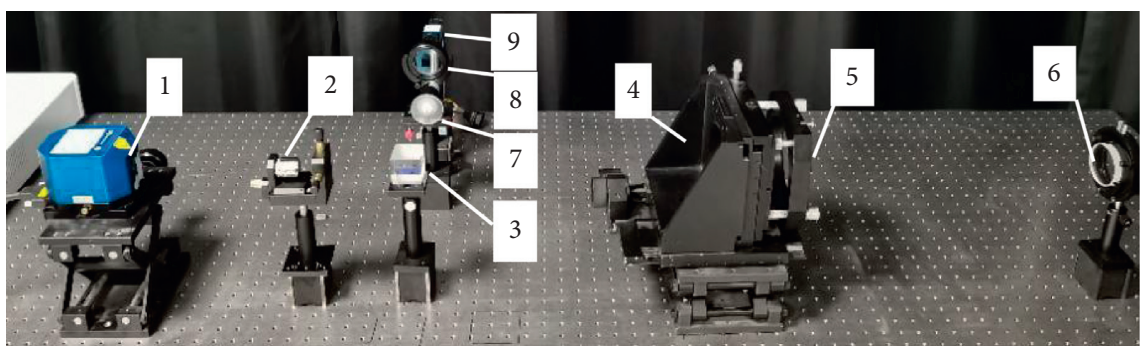

FiguRE 9: Experimental setup. 1, laser; 2, microscope objective; 3, beam splitter; 4, collimating lens; 5, reference flat; 6, testing flat; 7, diffuser; 8 , imaging lens 9-CCD.

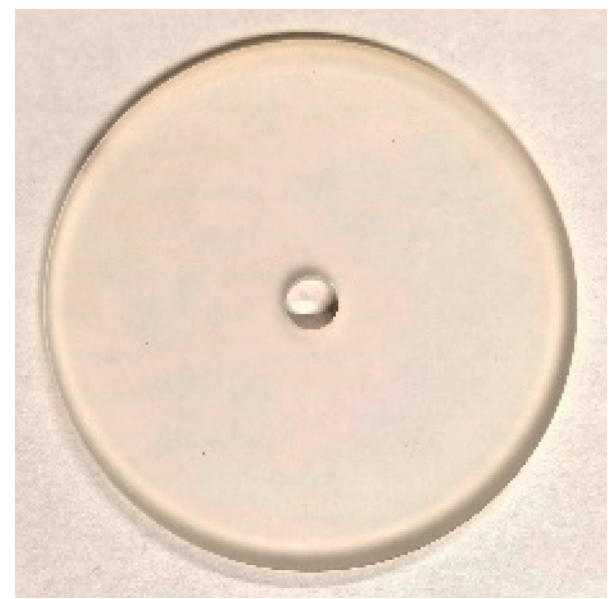

Figure 10: Diffuser.

TABLE 1: Diffuser with different surface roughnesses.

\begin{tabular}{lccc}
\hline$\sigma_{h} / \lambda$ & Designed RMS roughness $(\mathrm{nm})$ & Actual RMS roughness $(\mathrm{nm})$ & Error $(\mathrm{nm})$ \\
\hline 0.2 & 126.56 & 135.9 & 9.34 \\
0.3 & 189.84 & 199.8 & 9.96 \\
0.35 & 221.48 & 216.7 & -4.83 \\
0.4 & 253.12 & 244.4 & -8.7 \\
0.47 & 297.4 & 300.3 & 2.9 \\
0.5 & 316.4 & 305.1 & -11.3 \\
0.6 & 379.68 & 390.3 & 10.62 \\
0.7 & 442.96 & 458.7 & 15.74 \\
\hline
\end{tabular}

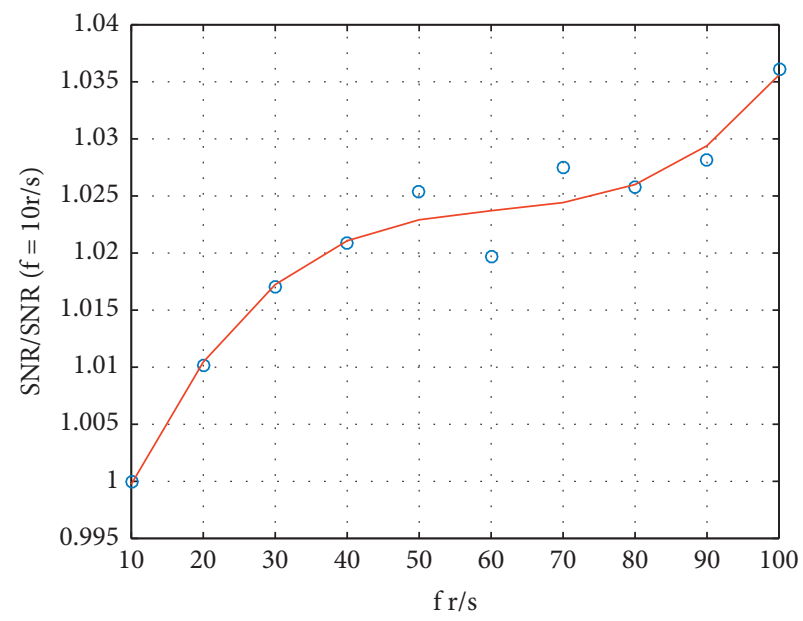

(a)

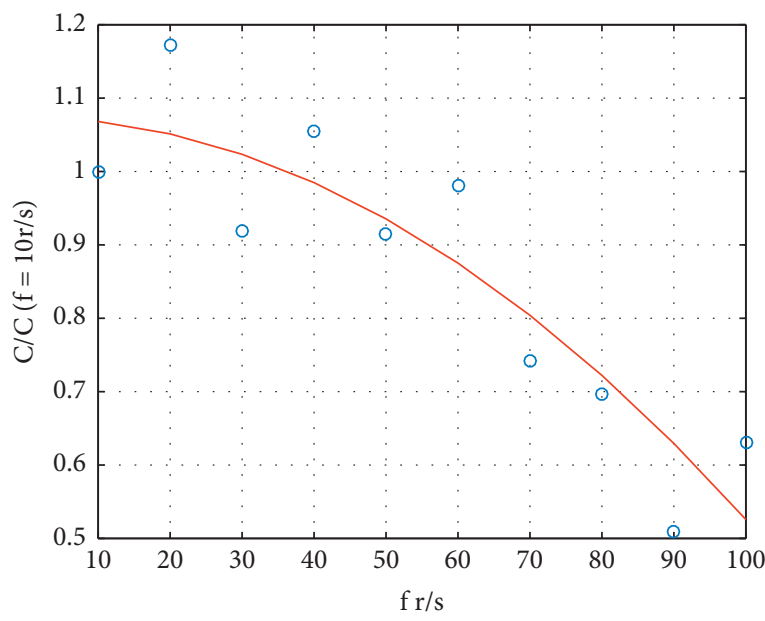

(b)

Figure 11: Continued. 


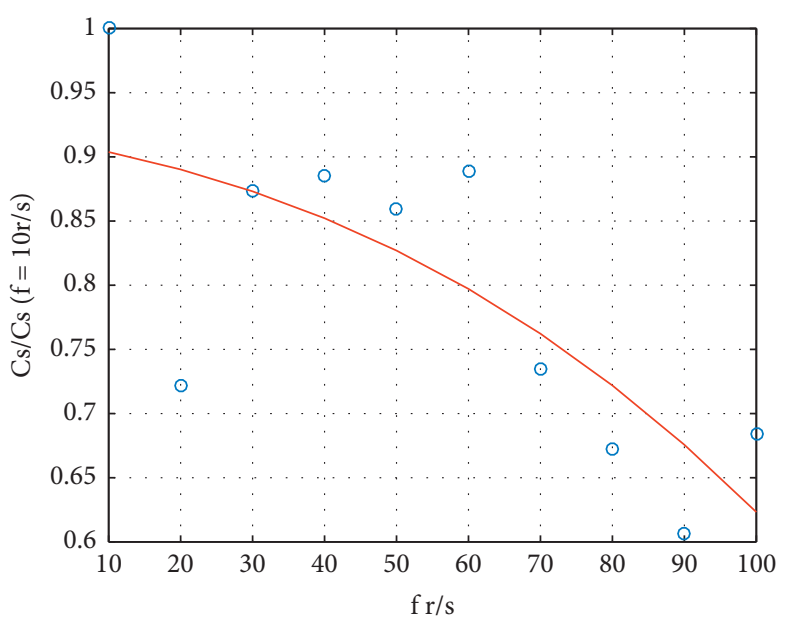

(c)

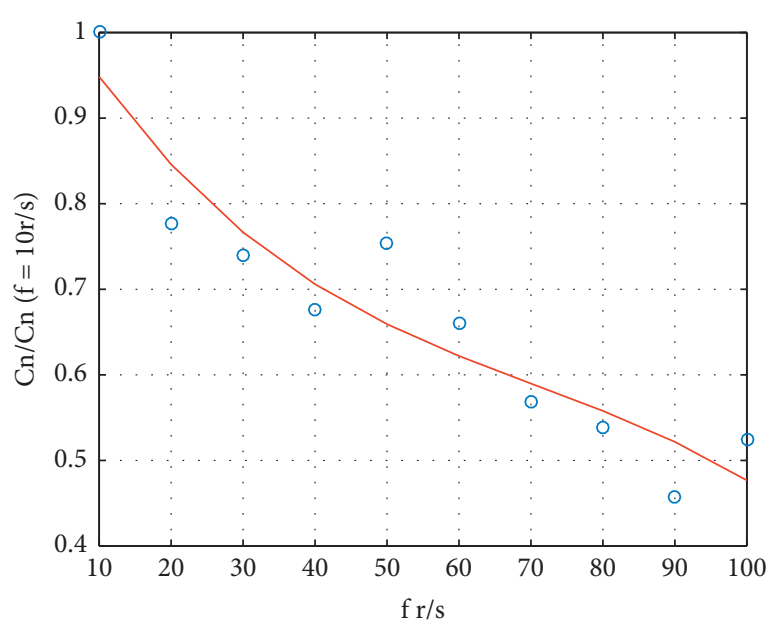

(d)

FIGURE 11: The relationship between the rotated diffuser and the parameters. (a) The influence on the SNR of the image. (b) The influence on the image contrast. (c) The influence on the contrast of the fringes. (d) The influence on the speckle contrast.

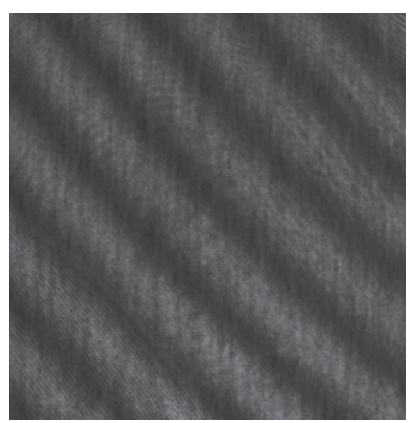

(a)

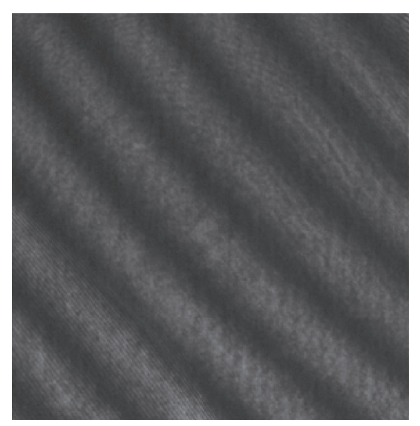

(e)

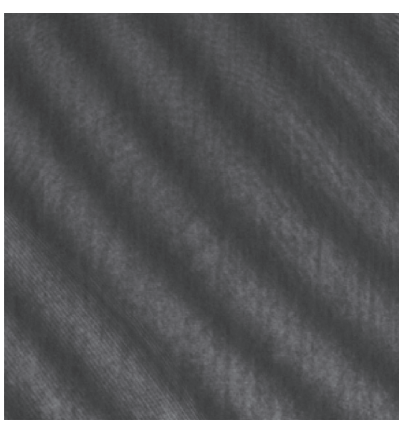

(b)

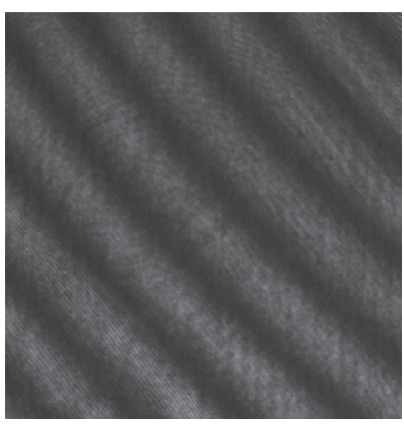

(f)

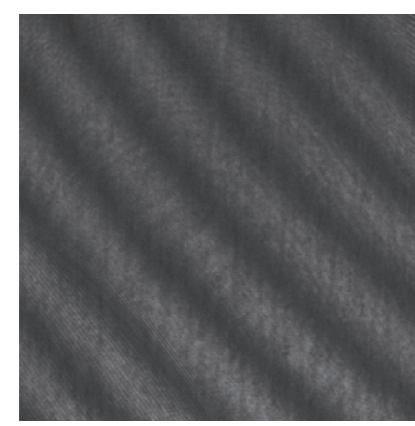

(c)

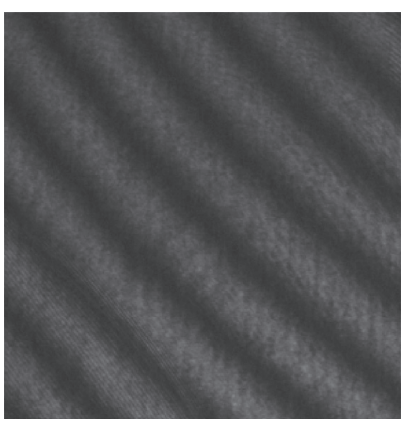

(g)

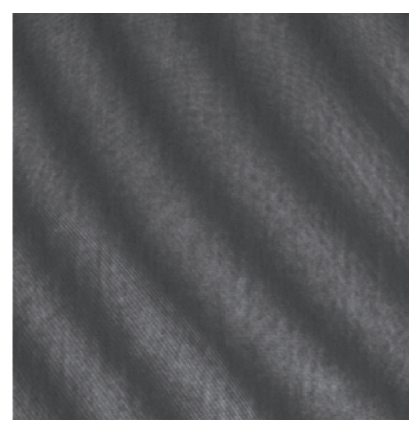

(d)

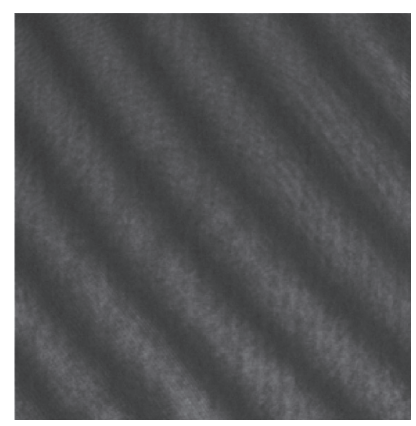

(h)

Figure 12: The interference image for different speeds of the rotated diffuser. (a) $f=10 \mathrm{r} / \mathrm{s}$. (b) $f=20 \mathrm{r} / \mathrm{s}$. (c) $f=30 \mathrm{r} / \mathrm{s}$. (d) $f=40 \mathrm{r} / \mathrm{s}$. (e) $f=50 \mathrm{r} / \mathrm{s}$. (f) $f=60 \mathrm{r} / \mathrm{s}$. (g) $f=70 \mathrm{r} / \mathrm{s}$. (h) $f=80 \mathrm{r} / \mathrm{s}$.

between 0.2 and 0.6 . When the rotation speed was $100 \mathrm{r} / \mathrm{s}$, the effective range of $\sigma_{h} / \lambda$ was between 0.4 and 0.6. The smaller the value of $\sigma_{h} / \lambda$, the smaller the contrast of the noise and the less obvious the effect of the noise reduction. However, when $\sigma_{h} / \lambda$ was bigger, the surface scattering characteristics of the diffuser were better. The entire light field was full of noise, which had no function of restraining noise but did seriously affect the quality of the interference image. There was a large error between the actual surface roughness and the designed surface roughness of the diffuser used in the experiment, leading to a large error in the numerical value between the experimental result and the 


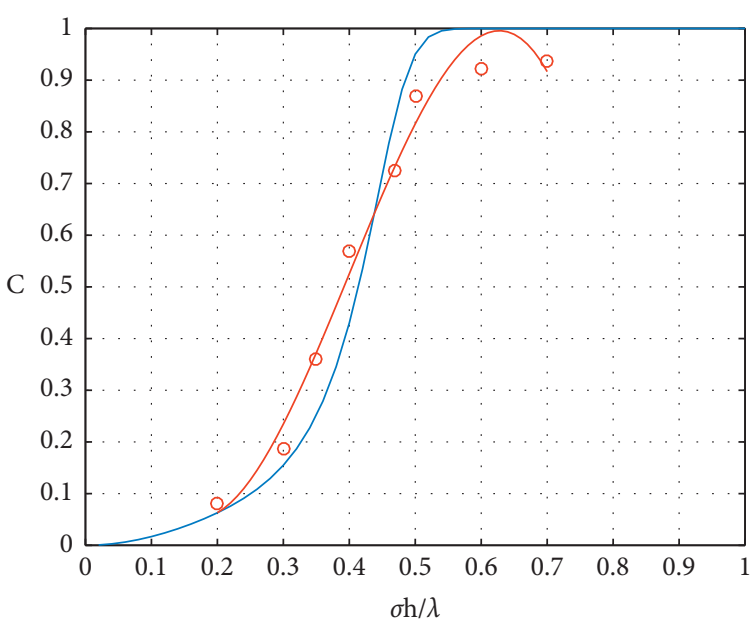

- Theoretical noise contrast

- Experimental noise contrast

_ Curve fitting of experimental noise contrast

(a)

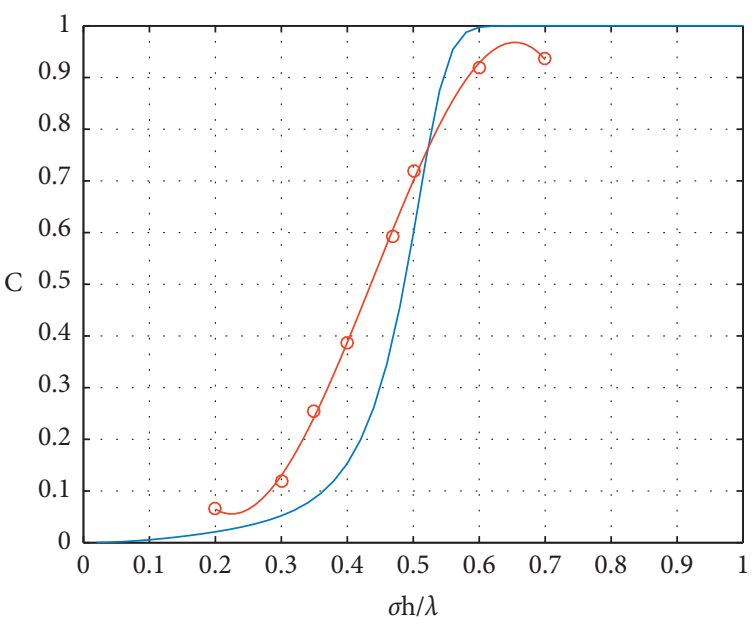

- Theoretical noise contrast

- Experimental noise contrast

_ Curve fitting of experimental noise contrast

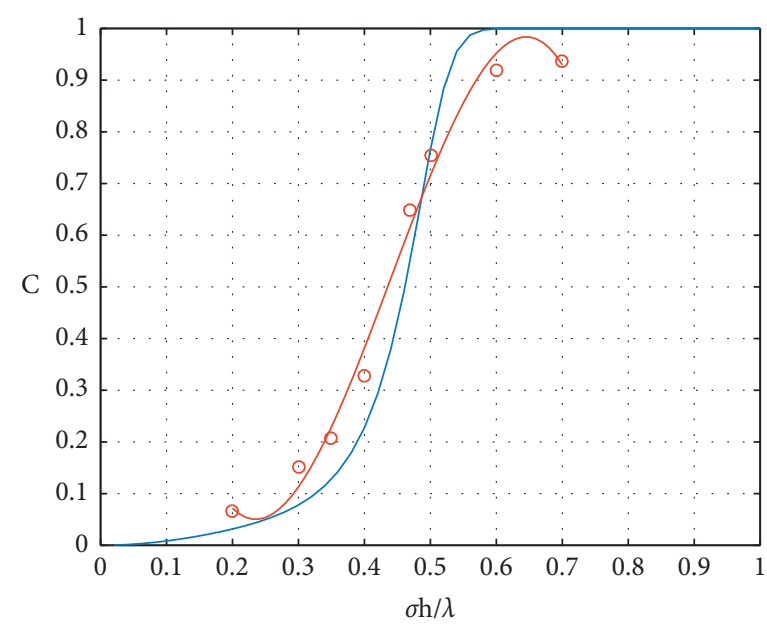

- Theoretical noise contrast

- Experimental noise contrast

_ Curve fitting of experimental noise contrast

(b)

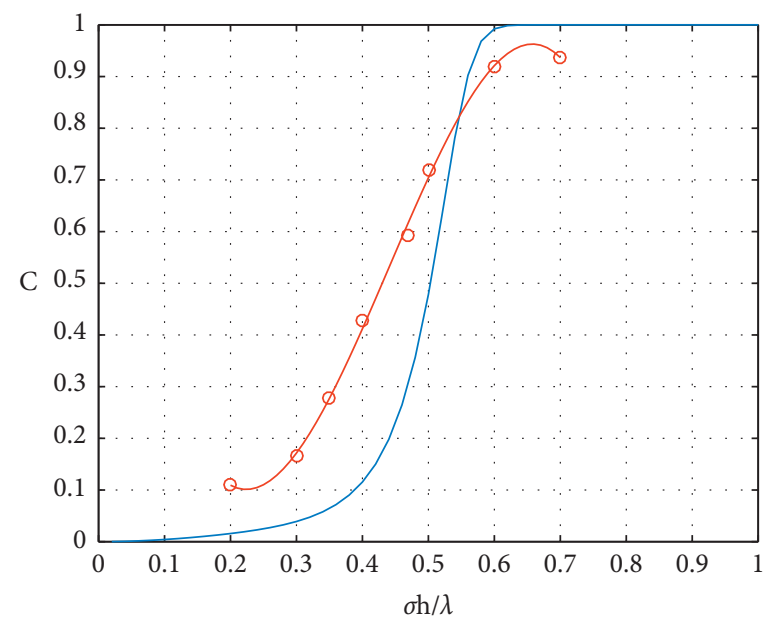

— Theoretical noise contrast

- Experimental noise contrast

_ Curve fitting of experimental noise contrast

(c)

Figure 13: Continued. 


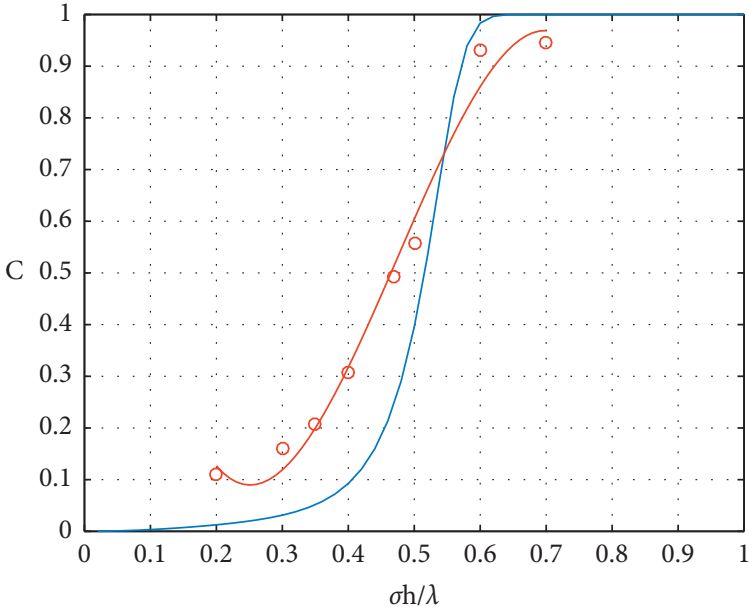

- Theoretical noise contrast

- Experimental noise contrast

_ Curve fitting of experimental noise contrast

(e)

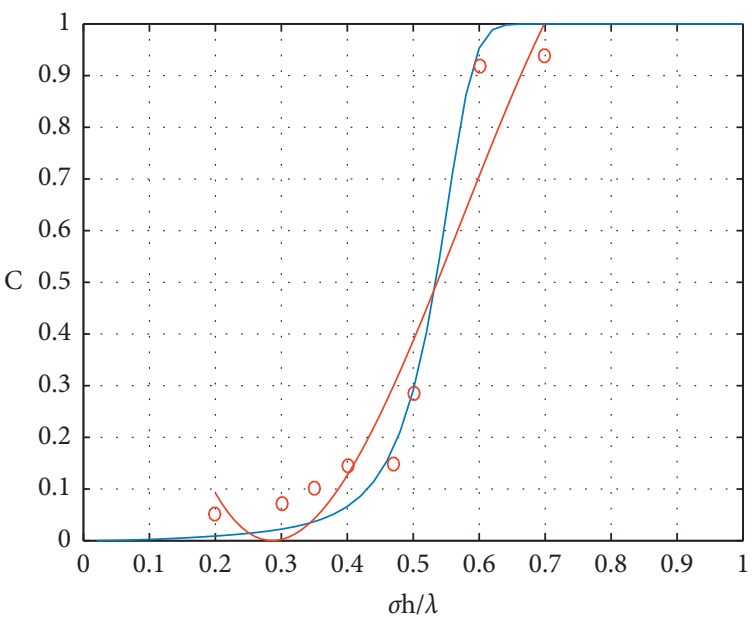

- Theoretical noise contrast

- Experimental noise contrast

_ Curve fitting of experimental noise contrast

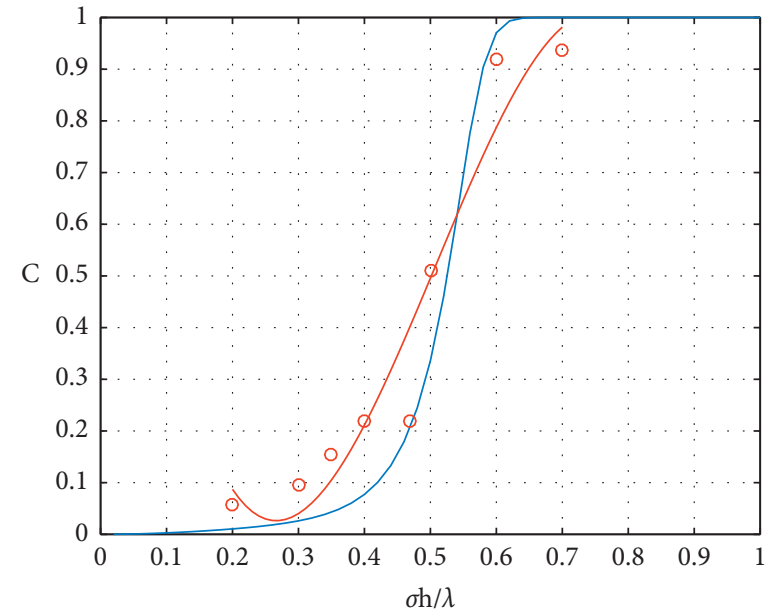

Theoretical noise contrast

- Experimental noise contrast

_ Curve fitting of experimental noise contrast

(f)

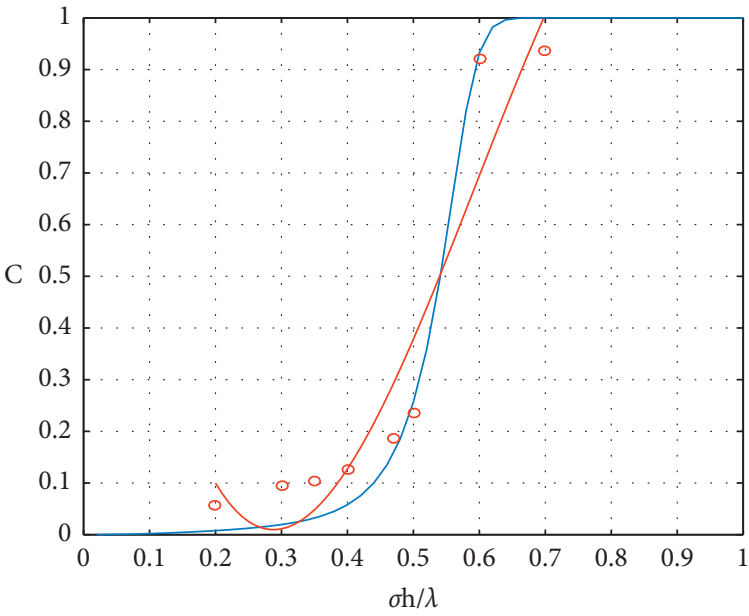

- Theoretical noise contrast

- Experimental noise contrast

_ Curve fitting of experimental noise contrast

(g)

Figure 13: Continued. 


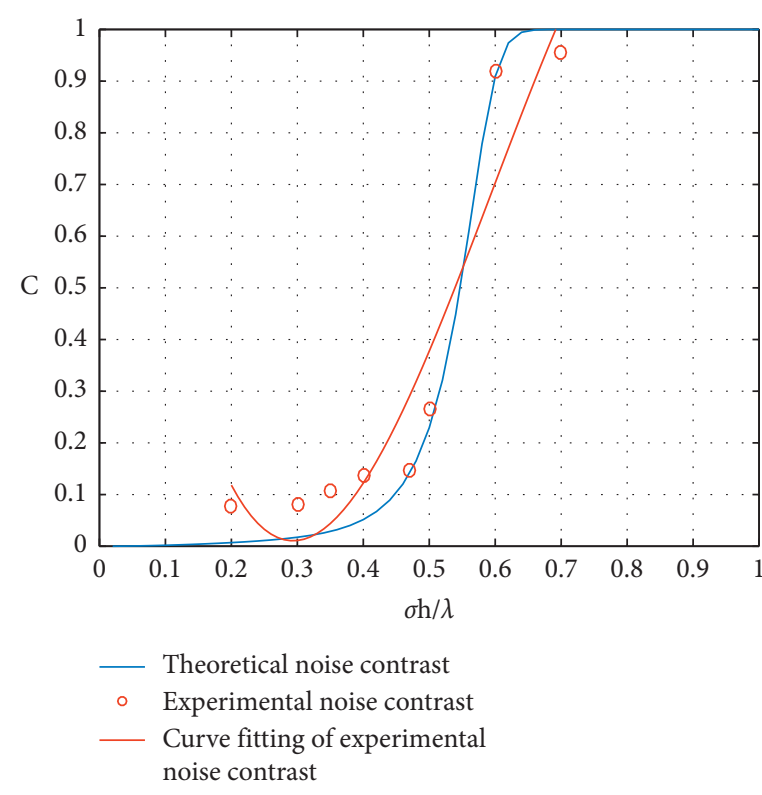

(i)

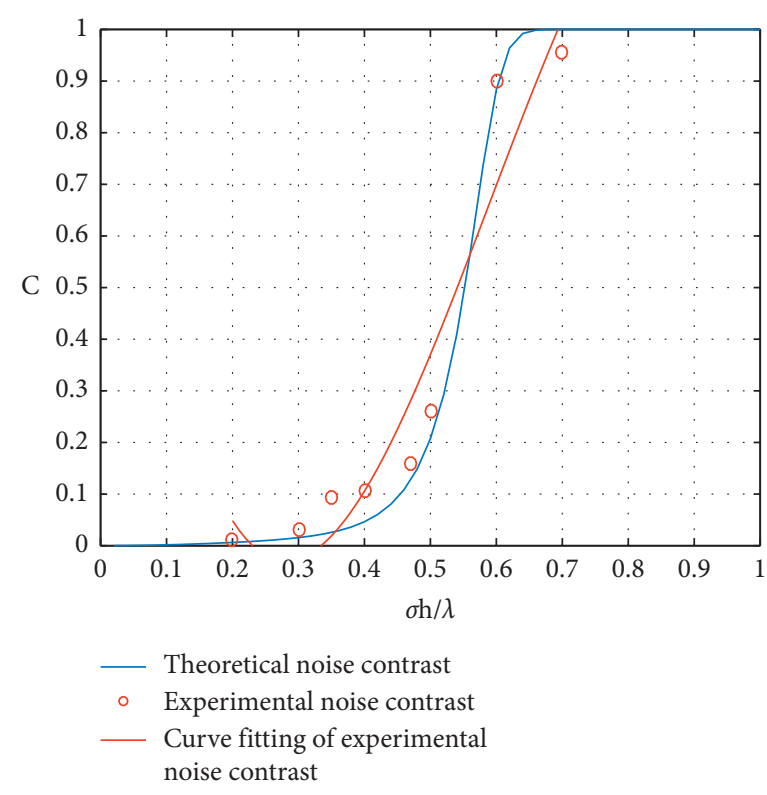

(j)

FIGURE 13: Effect of the surface roughness on the noise contrast at different rotational speeds. (a) V=10 r/s. (b) V=20 r/s. (c) V=30 r/s. (d) $\mathrm{V}=40 \mathrm{r} / \mathrm{s}$. (e) $\mathrm{V}=50 \mathrm{r} / \mathrm{s}$. (f) $\mathrm{V}=60 \mathrm{r} / \mathrm{s}$. (g) $\mathrm{V}=70 \mathrm{r} / \mathrm{s}$. (h) $\mathrm{V}=80 \mathrm{r} / \mathrm{s}$. (i) $\mathrm{V}=90 \mathrm{r} / \mathrm{s}$. (j) $\mathrm{V}=100 \mathrm{r} / \mathrm{s}$.

simulation result. However, the experimental result was generally consistent with the simulation result, with a good consistency.

\section{Conclusion}

In this research, the relationship between the rotating speed of the diffuser in the Fizeau interferometer and the contrast of the interference fringes, speckle contrast, image contrast, SNR, and coherent time was analyzed and verified with experiments. The experimental results showed that the noise of the interference system was obviously suppressed by adding the rotating diffuser in the interference system. At the same time, the fringe contrast of the interference image decreased with the increase of rotational speed, and the image signal-to-noise ratio was improved, which showed the consistency of the experimental results and the simulation results. Therefore, when a rotating diffuser was used to reduce the noise of the interference system, we had to choose the appropriate surface roughness and the matching rotation speed. The noise contrast ratio had to be properly reduced so that the quality of the interference image could be optimized.

\section{Data Availability}

The simulation analysis data used to support the findings of this study are included within the article. The experimental measurement data used to support the findings of this study are included within the article.

\section{Conflicts of Interest}

The authors declare that they have no conflicts of interest.

\section{Acknowledgments}

This research was financially supported by the China Research Project (Grant no. JSZL2018411C001) and Shaanxi Science and Technology Department Research Project (Grant no. 2020GY-045). The authors thank LetPub (http:// www.letpub.com) for its linguistic assistance during the preparation of this manuscript.

\section{References}

[1] G. Huang, Research on Fizeau Phase-Shifting Laser interferometer, Harbin Institute of Technology, Harbin, China, 2011.

[2] M. Kuechel, "Apparatus and method for reducing the effects of coherent artifact in an interferometer," United Stated patents: 6804011, 2004.

[3] Z. Chen, J. Li, and Y. Chen, "A practical method to suppress laser speckle noise in coherent imaging system," Chinese Journal of Lasers, vol. 12, no. 12, pp. 726-728, 1985.

[4] W. Wu, LRCS of Absorbing Coating Target under Coherent and Partially Coherent Light, XIdian University, Xi'an, China, 2014.

[5] T. Asakura, "Spatial coherence of laser light passed through rotating ground glass," Opto-electronics, vol. 2, no. 3, pp. 115-123, 1970.

[6] O. Kwon, J. C. Wyant, and C. R. Hayslett, "Rough surface interferometry at $10.6 \mu \mathrm{m}$," Applied Optics, vol. 19, no. 11, pp. 1862-1869, 1968.

[7] V. Harwalkar, H. Bohidar, and S. Chopra, "Theoretical and experimental investigations of light scattered from a rotating ground glass," Applied Physics B Photophysics and Laser Chemistry, vol. 31, no. 4, pp. 215-220, 1983.

[8] L. Chen, Research on Infrared Fizeau Interferometer and Automatic Infrared Interferogram Processing, Nanjing University of Science \& Technology, Nanjing, China, 1996. 
[9] M. Ziraki, R. Jones, P. M. W. French et al., "Whole-field coherent imaging through turbid media using Photorefractive holography]," Applied Physics B, vol. 70, no. 1, pp. 151-154, 2000.

[10] M. C. Pitter, C. W. See, and M. G. Somekh, "Full-field heterodyne interference microscope with spatially incoherent illumination," Optics Letters, vol. 29, no. 11, pp. 1200-1202, 2004.

[11] M. N. Morris, M. Naradikian, and J. Millerd, "Noise Reduction in dynamic interferometery measurements," Interferometry XV: Techniques and Analysis, vol. 7790, Article ID 77900, 2010.

[12] J. Xu, Z. Liu, Y. Du et al., "Statistical analysis of interferometric imaging system with rotating diffuser," High Power Laser and Particle Beams, vol. 23, no. 3, pp. 702-706, 2011.

[13] C.-Y. Chen, W.-C. Su, C.-H. Lin, M.-D. Ke, Q.-L. Deng, and K.-Y. Chiu, "Reduction of speckles and distortion in projection system by using a rotating diffuser," Optical Review, vol. 19, no. 6, pp. 440-443, 2012.

[14] T. Qiao, Y. Yu, and W. Zhou, "A method to eliminate the noise of holographic system by using a rotated diffuser," Optical Instruments, vol. 36, no. 4, pp. 337-341, 2014.

[15] J. W. Goodman, Speckle Phenomena in Optics-Theory and Application, Roberts \& Company, Aurora, Canada, 2007. 